https://doi.org/10.15407/ujpe66.7.582

L.A. NAJAM, ${ }^{1}$ S.N. ABOOD ${ }^{2}$ F.M.A. AL-JOMAILY ${ }^{1}$

${ }^{1}$ Dept. of Physics, College of Science, Mosul University

(Mosul, Iraq; e-mail: prof.lai2014@uomosul.edu.iq)

2 Dept. of Physics, College of Science, Al-Nahrain University

(Baghdad, Iraq; e-mail: saadnaji@yahoo.com)

\title{
CONFIGURATION MIXING FOR Po ISOTOPES WITHIN THE INTERACTING BOSON MODEL-2
}

\begin{abstract}
We analyze a sequence of ${ }^{194-204}$ Po isotopes, using the Configuration Mixing (CM) Interacting Boson Model 2 (IBM-2). We set the parameters of Hamiltonian using a least-square fit for the known energy levels, electrical transition rates $B(E 2)$, and quadruple moments $Q\left(2_{1}^{+}\right)$for the first excited states. We have a good agreement with the experimental values for all the observables tested, and we infer that the feature of the shape coexistence is concealed in the isotopes of Po, just as in the isotopes of Os and Pt.

Ke ywords: Interacting Boson Model (IBM-2), configuration mixing, $B(E 2), Q\left(2_{1}^{+}\right)$.
\end{abstract}

\section{Introduction}

The concept of shape coexistence is very well known in the area of lead isotopes, as well as in a variety of other shell closures, and was examined by Wood et al., [1]. However, the clear practical evidence of the texture and evolution of existing configurations around the shape is mostly limited to the isotopes of $\mathrm{Pb}, \mathrm{Hg}$, and $\mathrm{Pt}$. The $\mathrm{Pt}$ and $\mathrm{Hg}$ isotopes were analyzed in $[2,3]$ and [4], respectively, and $\mathrm{Yb}-\mathrm{Pt}$ nuclei with $N=108$ were considered in [5]. Oros et al. [6] used the particle-core model to study the shape coexistence in light Po isotopes, and GarciaRamos and Heyde [7] considered the nuclear shape coexistence in Po isotopes within the interacting boson model.They found that the lead zone, $\mathrm{Po}, \mathrm{Pb}$, $\mathrm{Hg}$, and $\mathrm{Pt}$ have coexisting structures with different deformations and correspond to different shellmodel particle-hole configurations. If the $0_{2}^{+}$states in the polonium isotopes arise from a second configuration $\left(N_{\pi}=3\right)$, we can assume that the IBM2 with the configuration mixing formalism describes

(C) L.A. NAJAM, S.N. ABOOD, F.M.A. AL-JOMAILY, 2021 the nuclear structure of Po isotopes. This model imples that the medium and heavy even-even nuclei can be represented by the inert core of valence nucleons whose pairs interact weakly with each other. The purpose of this work is to assess the importance of the configuration mixing in comprehending the nuclear structure of Po isotopes within IBM-2 CM, where the phenomena of shape coexistence is not sufficiently clear.

\section{Theoretical Framework}

The IBM-2 $[7,8]$ is a rather complex ideology in the physics of nuclear structure. It focuses on group theory principles and the classification of coupled nucleons as bosons. The second incarnation of the theory labeled IBM-2, in which protons and neutrons are distinguished, is discussed in what follows. In the context of IBM-2, nucleons with a spin of either 0 or 2 are categorized as $s$ and $d$-bosons and are considered to be coupled into bosons. In the group theory language, the key collective nucleus motions are characterized using the sets, U(5) (anharmonic vibrator), $\mathrm{SU}(3)$ (symmetric rotor), and $\mathrm{O}(6)$ ( $\gamma$-soft

ISSN 2071-0194. Ukr. J. Phys. 2021. Vol. 66, No. 7 
rotor). The IBM-2 Hamiltonian is given by [8]:

$H=\varepsilon\left(\hat{n}_{d s}+\hat{n}_{d \nu}\right)+K Q_{\pi} Q_{\nu}+K\left(Q_{\nu} \cdot Q_{\pi}+Q_{\pi} \cdot Q_{\nu}\right)+$

$+V_{\pi \pi}+V_{\nu \nu}+M_{\pi \nu}$,

where $Q_{\rho}$ is the operator of quadrupole moment:

$Q_{\pi}=\left[d^{+} \times S^{\sim}+S^{+} \times d^{\sim}\right]^{(2)}+\chi_{\pi}\left[d^{+} \times d^{\sim}\right]$

and

$Q_{\nu}=\left[d^{+} \times S^{\sim}+S^{+} \times d^{\sim}\right]^{(2)}+\chi_{\nu}\left[d^{+} \times d^{\sim}\right]$,

$\rho$ is the energy of a $d$-boson, $k$ is the strength of the quadrupole-quadrupole interaction between neutron bosons and proton bosons, and $\chi_{\rho}$ is the quadrupole deformation parameter for bosons that determines the sign of the operator of quadrupole moment. The term $V_{\pi \pi}$ is the interaction of proton bosons, and $V_{\nu \nu}$ is the interaction of neutron bosons only. They are given by [8]:

$V_{\pi \pi}=\frac{1}{2} \sqrt{2 L+1} \sum_{L=0,2,4} C_{L \pi}\left[\left(d_{\pi}^{+} d_{\pi}^{+}\right)^{(L)}\left(d_{\pi}^{+} d_{\pi}^{\sim}\right)\right]^{(0)}$

and

$V_{\nu \nu}=\frac{1}{2} \sqrt{2 L+1} \sum_{L=0,2,4} C_{L \nu}\left[\left(d_{\nu}^{+} d_{\nu}^{+}\right)^{(L)}\left(d_{\nu}^{+} d_{\nu}^{\sim}\right)\right]^{(0)}$.

Finally, the term $M_{\pi \nu}$ is the Majorana force parameter:

$$
\begin{aligned}
& M_{\pi \nu}=-\sum_{k=1-3} 2 \xi_{k}\left(d_{\pi}^{+} \times d_{\pi}^{+}\right)^{(k)}\left(d_{\pi}^{\sim} \times d_{\pi}^{\sim}\right)^{(k)}+ \\
& +\xi_{2}\left(d_{\pi}^{+} \times S_{\nu}^{+}-S_{\pi}^{\sim} \times d_{\nu}^{\sim}\right)^{(2)}\left(d_{\pi}^{\sim} \times S_{\nu}^{\sim}-S_{\pi}^{\sim} \times d_{\pi}^{\sim}\right)^{(2)} .
\end{aligned}
$$

The IBM-2 treats the configuration mixing by using the technique developed by Duval and Barrett [10]. The calculations within IBM-2 CM are separated for two configurations, and the results are then mixed using their interaction.

The Interacting Boson Model (IBM-2) [8] describes the nuclear shape coexistence phenomenon and considers that the excitation levels of the isotope are characterized by the number of bosons. The bosons are coupled with the angular momentum $L=0$ or $L=2$ for a given nucleus, where $N$ is constant. The IBM-2 system of configurations with $N, N+2, N+4$, $N+6, \ldots$ corresponds to the configuration mixing in the shell model " $0 p-0 h, 2 p-2 h, 4 p-4 h, 6 p-6 h, \ldots$ ". The IBM-2 has ability to treat this case in the configuration mixing (IBM-2 CM) [11]; the application of this model was pointed out by Fossion et al., [12] for $\mathrm{Pb}$ nuclei.

The two configurations are connected in the Hamiltonian called a mixing Hamiltonian, which is given in the form [10]:

$H_{\text {mix }}=H_{1}+H_{2}+V_{\text {mix }}$

where $H_{1}$ is the Hamiltonian of IBM-2 for the first configuration $\left(N_{\pi}=1\right), H_{2}$ is the Hamiltonian of IBM-2 for the second configuration $\left(N_{\pi}=3\right)$, which is given in Eq. (1), and $V_{\text {mix }}$ is the interaction mixing parameter for two configurations:

$V_{\text {mix }}=\alpha\left(S_{\pi}^{+} \times S_{\pi}^{+}+S_{\pi}^{\sim} \times S_{\pi}^{\sim}\right)^{(0)}+$

$+\beta\left(d_{\pi}^{+} \times d_{\pi}^{+}+d_{\pi}^{\sim} \times d_{\pi}^{\sim}\right)^{(0)}$

The normal configuration consists of two protons (one boson $N_{\pi}=1$ ), while the intruder configuration (second configuration) is represented by $N_{\pi}=3$ with the excitations across the major shell $(Z=82)$. The parameters $\alpha$ and $\beta$ are called the mixing strength parameters. The third parameter $\Delta$ is the pair excitation energy of the bosons across the major shell and gives the relative energy of two configurations [10]. The transition operators in IBM-2 are defined as [8]:

$T^{(l)}=T_{\pi}^{(l)}+T_{\nu}^{(l)}$.

The operator of electric quadrupole transition takes the form:

$T^{(E 2)}=e_{\pi} Q_{\pi}^{\chi \pi}+e_{\nu} Q_{\nu}^{\chi \nu}$

where $e_{\pi}\left(e_{\nu}\right)$ are effective charges for proton (neutron) bosons, respectively. In the configuration mixing, the effective charges for the proton and neutron bosons are often considered equal for simplicity: $e_{\pi}=e_{\nu}=e$. Therefore, Eq. (8) becomes as:

$T^{(E 2)}=e\left(Q_{\pi}+Q_{\nu}\right)$

The electric quadrupole transition operator for two configurations is given by [11]:

$T^{(E 2)}=e_{1}\left[\left(Q_{1 \pi}+Q_{1 \nu}\right)+\left(\frac{e_{3}}{e_{2}}\right)\left(Q_{3 \pi}+Q_{3 \pi}\right)\right]$.

To evaluate the effective charges for proton and neutron bosons, we used the method in Ref. [13] and performed the fitting of the the experimental value of 
Table 1. IBM-2 variables of the Hamiltonian used in the calculations of Po isotopes. The $\chi$ and $s$ parameters are dimensionless; the other parameters are given in MeV

\begin{tabular}{|c|c|c|c|c|c|c|c|c|c|c|c|c|}
\hline Isotopes & $\varepsilon^{1 \pi}$ & $K^{1 \pi}$ & $\chi^{1 \pi}$ & $\chi^{1 \nu}$ & $C_{0 \nu}^{1 \pi}$ & $C_{2 \nu}^{1 \pi}$ & $\xi_{2}^{1 \pi}$ & $\xi_{1}^{1 \pi}=\xi_{3}^{1 \pi}$ & $\varepsilon^{3 \pi}$ & $\xi_{1}^{3 \pi}=\xi_{3}^{3 \pi}$ & $\alpha=\beta$ & $\Delta$ \\
\hline${ }^{192} \mathrm{Po}$ & 1.00 & -0.16 & 0.4 & -0.8 & 0.30 & 0.102 & 0.25 & 0.02 & 0.0 .33 & -0.085 & 0.125 & 4.20 \\
\hline${ }^{194} \mathrm{Po}$ & 1.62 & -0.15 & 0.4 & -1.0 & 0.28 & 0.102 & 0.25 & 0.02 & 0.0 .33 & -0.085 & 0.125 & 4.20 \\
\hline${ }^{196} \mathrm{Po}$ & 1.15 & -0.14 & 0.4 & -1.0 & 0.16 & 0.102 & 0.25 & 0.02 & 0.0 .33 & -0.085 & 0.125 & 4.20 \\
\hline${ }^{198} \mathrm{Po}$ & 0.90 & -0.14 & 0.4 & -1.2 & -0.28 & 0.102 & 0.25 & 0.02 & 0.0 .33 & -0.085 & 0.125 & 4.20 \\
\hline${ }^{200} \mathrm{Po}$ & 0.80 & -0.15 & 0.4 & -1.1 & -0.15 & 0.102 & 0.25 & 0.02 & 0.0 .33 & -0.085 & 0.125 & 4.20 \\
\hline${ }^{202} \mathrm{Po}$ & 0.70 & -0.155 & 0.4 & -1.1 & -0.12 & 0.102 & 0.25 & 0.02 & 0.0 .33 & -0.085 & 0.125 & 4.20 \\
\hline${ }^{204} \mathrm{Po}$ & 0.60 & -0.16 & 0.4 & -1.0 & -0.10 & 0.102 & 0.25 & 0.02 & 0.0 .33 & -0.085 & 0.125 & 4.20 \\
\hline
\end{tabular}

$C_{0 \nu}^{3 \pi}=C_{2 \nu}^{3 \pi}=C_{4 \nu}^{3 \pi}=0.0 \mathrm{MeV}, C_{0 \nu}^{3 \pi}=C_{0 \nu}^{1 \pi}=0.0 \mathrm{MeV}, \xi_{2}^{3 \pi}=0.0 \mathrm{MeV}$

$B\left(E 2 ; 2_{1}^{+} \rightarrow 0_{1}^{+}\right)$. The reduced electric transition rate can be written as [14]:

$B\left(E 2 ; I_{i}^{+} \rightarrow I_{f}^{+}\right)=\frac{\left|\left\langle I_{f}|| T^{(E 2)}|| I_{i}\right\rangle\right|}{2 I_{i}+1}$.

$\mathrm{r}$ The quadrupole moment for the fist excited state is as follows [14]:

$Q\left(2_{1}^{+}\right)=\left(\frac{32 \pi}{175}\right)^{1 / 2}\left\langle 2_{1}^{+}\left\|T^{(E 2)}\right\| 2_{1}^{+}\right\rangle$.

\section{Results and Discussion}

\subsection{Energy levels}

The parameters of the Hamiltonian for two configurations are given in Table 1 . One can observe that the $\varepsilon, k, \chi_{\nu}$ and $C_{o \nu}$ parameters in two configurations vary from isotope to isotope with increasing the neutron number; these parameters are free parameters. The Majorana force parameters $\xi_{1}=\xi_{2}$ are constants for all isotopes in two configurations. The other parameters are remaining fixed or give negligible contributions into the calculations. The pair excitation energy $\Delta$ is a function of the neutron number or the number of neutron bosons. Our $\Delta$ parameter values used in calculations for Po isotopes are larger than the values in [15], because we assume that the second configuration is based on one proton pair of $Z=82$ shell within the same valence space. The mixing parameters are taken $\alpha=\beta$ for all isotopes.

In Table 2, we show excitations of the first configuration $N_{\pi}=1$, as well as the intruder excitations. The systematic is locally disturbed by the mixing between regular and intruder configurations. This becomes even more clear, when looking at the $I=2$ multiple involving the regular or normal configuration of ${ }^{184-192}$ Os isotopes $\left(N_{\pi}=3\right)$ and ${ }^{192-204} \mathrm{Po}$ isotopes $\left(N_{\pi}=3\right)$ intruder excitations. In Table 1, Po isotopes for $N_{\pi}=1$ lie in vibrational nuclei near the spherical shape, and these isotopes tend to a deformed shape.

In Table 3, we show the comparison of the data for ${ }^{192-204} \mathrm{Po}$ isotopes (intruder configuration $N_{\pi}=3$ ) and ${ }^{184-192}$ Os isotopes (normal configuration $N_{\pi}=$ $=3$ ), where the intruder energies have been reconstructed [16-20]. It is clear that the Po isotopes in the intruder configuration $N_{\pi}=3$ have the same behavior as ${ }^{184-192}$ Os isotopes.

There are, however, emerging discrepancies near the paring gap at $N_{\pi}=3$ which may be the asymptotics of the admixture of non-collective excitations which are outside the IBM-2 space, In particular, the highest spin states (higher band) is too high in energy. In addition, our calculations predict the band $K^{\pi}=4^{+}$at about $3.0 \mathrm{MeV}$ for ${ }^{200} \mathrm{Po}$. The experimental behavior of $0+$ states is consistent with this IBM$2 \mathrm{CM}$ picture. The energy of the $0_{2}^{+}$states (intruder states) increases, in fact, with the number of neutrons and goes up in two configurations, although few experimental information about intruder $0_{2}^{+}$states is available $[22,23]$.

\subsection{Electric Transition Probability B(E2)}

The reduced electric transition rates which are given in Eq. (10) depend on the effective charges for bosons, $e_{\pi}$ and $e_{v}$. From the energy levels in Table 2, the ratio $E\left(4_{1}^{+}\right) / E\left(2_{1}^{+}\right)$for ${ }^{194-202}$ Po isotopes equals about 2 , which means that the Po isotopes are characterized by the $\mathrm{U}(5)$ vibrational symmetry and possess an almost spherical shape. Therefore, we use this sym- 
Table 2. Energy levels for Po isotopes in $\mathrm{MeV}$ units for the configuration $\boldsymbol{N}_{\boldsymbol{\pi}}=1$

\begin{tabular}{|c|c|c|c|c|c|c|c|c|c|c|c|c|}
\hline \multirow{2}{*}{$2^{*} J_{i}^{+}$} & \multicolumn{2}{|c|}{${ }^{194} \mathrm{Po}$} & \multicolumn{2}{|c|}{${ }^{196} \mathrm{Po}$} & \multicolumn{2}{|c|}{${ }^{198} \mathrm{Po}$} & \multicolumn{2}{|c|}{${ }^{200} \mathrm{Po}$} & \multicolumn{2}{|c|}{${ }^{202} \mathrm{Po}$} & \multicolumn{2}{|c|}{${ }^{204} \mathrm{Po}$} \\
\hline & Exp. & IBM-2 & Exp. & IBM-2 & Exp. & IBM-2 & Exp. & IBM-2 & Exp. & IBM-2 & Exp. & IBM-2 \\
\hline $0_{1}$ & 0.00 & 0.00 & 0.00 & 0.00 & 0.00 & 0.00 & 0.00 & 0.00 & 0.00 & 0.00 & 0.00 & 0.00 \\
\hline $2_{1}$ & $0.319(3)$ & 0.319 & $0.463(6)$ & 0.464 & $0.605(10)$ & 0.605 & $0.666(14)$ & 0.665 & $0.677(20)$ & 0.665 & $0.684(20)$ & 0.684 \\
\hline $4_{1}$ & $0.684(4)$ & 0.683 & $0.891(12)$ & 0.889 & $1.158(13)$ & 1.160 & - & 1.179 & $1.248(3)$ & 1.249 & $1.200(3)$ & 1.202 \\
\hline $2_{2}$ & $0.659(4)$ & 0.670 & $0.859(11)$ & 0.844 & $1.039(14)$ & 1.002 & - & 1.032 & - & 1.143 & $1.255(3)$ & 1.267 \\
\hline $3_{1}$ & - & 0.789 & - & 0.983 & - & 0.997 & - & -1.001 & - & 1.110 & - & 1.230 \\
\hline $\mathrm{O}_{2}$ & - & 0.543 & $0.558(1)$ & 0.600 & $0.815(10)$ & 0.845 & $1.137(9)$ & 1.198 & - & 1.201 & - & 1.254 \\
\hline $4_{2}$ & $1.209(5)$ & 1.30 & $0.891(13)$ & 0.901 & $1.483(16)$ & 1.532 & $1.277(19)$ & 1.340 & $1.773(3)$ & 1.821 & $1.552(6)$ & 1.590 \\
\hline 61 & $1.146(5)$ & 1.160 & $1.390(13)$ & 1.397 & $1.717(18)$ & 1.632 & $1.762(10)$ & 1.776 & $1.691(4)$ & 1.700 & $1.626(4)$ & 1.720 \\
\hline $2_{3}$ & $1.146(3)$ & 1.231 & $1.039(2)$ & 1.00 & - & 1.380 & $1.392(9)$ & 1.420 & - & 1.521 & - & 1.610 \\
\hline $8_{1}$ & $1.690(6)$ & 1.567 & $1.939(19)$ & 1.889 & $1.835(18)$ & 1.929 & - & 1.998 & $1.772(5)$ & 1.802 & $1.683(5)$ & 1.731 \\
\hline $4_{3}$ & - & 1.228 & $1.388(2)$ & 1.301 & - & 1.420 & $1.773(12)$ & 1.860 & - & 1.875 & - & 1.899 \\
\hline
\end{tabular}

Experimental data are given from Refs [24-26].

Table 3. Energy levels for Po isotopes in MeV units for the configuration $\boldsymbol{N}_{\boldsymbol{\pi}}=\mathbf{3}$

\begin{tabular}{|c|c|c|c|c|c|c|c|c|c|c|}
\hline \multirow{2}{*}{$2^{*} J_{i}^{\pi}$} & \multicolumn{2}{|c|}{$N_{\nu}=9$} & \multicolumn{2}{|c|}{$N_{\nu}=8$} & \multicolumn{2}{|c|}{$N_{\nu}=7$} & \multicolumn{2}{|c|}{$N_{\nu}=6$} & \multicolumn{2}{|c|}{$N_{\nu}=5$} \\
\hline & ${ }^{184} \mathrm{Os}$ & ${ }^{192} \mathrm{Po}$ & ${ }^{186} \mathrm{Os}$ & ${ }^{194} \mathrm{Po}$ & ${ }^{188} \mathrm{Os}$ & ${ }^{196} \mathrm{Po}$ & ${ }^{190} \mathrm{Os}$ & ${ }^{198} \mathrm{Po}$ & ${ }^{192} \mathrm{Os}$ & ${ }^{200} \mathrm{Po}$ \\
\hline $0_{1}$ & 0.0 & 0.0 & 0.0 & 0.0 & 0.0 & 0.0 & 0.0 & 0.0 & 0.0 & 0.0 \\
\hline $2_{1}$ & 0.334 & 0.267 & 0.379 & 0.359 & 0.671 & 0.791 & 0.793 & 0.851 & 1.119 & 1.172 \\
\hline $\mathrm{O}_{2}$ & 0.732 & 0.742 & 0.567 & 0.889 & 0.632 & 0.921 & 0.932 & 1.393 & 1.321 & 1.407 \\
\hline $4_{1}$ & 0.997 & 0.998 & 0.681 & 1.191 & 1.036 & 1.324 & 1.402 & 1.862 & 1.738 & 1.824 \\
\hline $6_{1}$ & 1.479 & 1.530 & 1.136 & 1.474 & 1.500 & 1.637 & 1.885 & 2.1191 & 2.209 & 2.456 \\
\hline $8_{1}$ & 1.493 & 2.187 & 1.672 & 2.270 & 2.081 & 2.229 & 2.541 & 2.750 & 2.881 & 3.889 \\
\hline
\end{tabular}

Table 4. Electric transition probabilities

(in $e$ $2 b 2$ units) and quadrupole moments (in $e b$ units) for Po isotopes

\begin{tabular}{|l|l|l|l|l|c|}
\hline \multirow{2}{*}{$2^{*} B\left(E 2 ; J_{i}^{+} \rightarrow J_{f}^{+}\right)$} & ${ }^{194} \mathrm{Po}$ & ${ }^{196} \mathrm{Po}$ & ${ }^{198} \mathrm{Po}$ & ${ }^{200} \mathrm{Po}$ & ${ }^{202} \mathrm{Po}{ }^{200}$ \\
\cline { 2 - 6 } & IBM-2 & IBM-2 & IBM-2 & IBM-2 & IBM-2 \\
\hline$B\left(E 2 ; 2_{1}^{+} \rightarrow 0_{1}^{+}\right)$ & 0.00203 & 0.00345 & 0.00520 & 0.00723 & 0.00951 \\
$B\left(E 2 ; 4_{1}^{+} \rightarrow 2_{1}^{+}\right)$ & 0.0121 & 0.0325 & 0.0342 & 0.0431 & 0.0987 \\
$B\left(E 2 ; 2_{2}^{+} \rightarrow 2_{1}^{+}\right)$ & 0.0166 & 0.0283 & 0.0406 & 0.0500 & 0.0478 \\
$B\left(E 2 ; 2_{3}^{+} \rightarrow 2_{1}^{+}\right)$ & 0.0012 & 0.0023 & 0.0498 & 0.0550 & 0.0470 \\
$Q\left(2_{1}^{+}\right)$ & 1.633 & 1.543 & 1.420 & 1.38 & 0.0610 \\
\end{tabular}

metry to estimate the effective charges for bosons [22]. These effective charges for bosons are normalized to experimental values of $B\left(E 2 ; 2_{1}^{+} \rightarrow 0_{1}^{+}\right)$. For simplicity, we use $e_{\pi}=e_{v}=e_{1}$, where $e_{1}=0.29 \mathrm{eb}$ and $e_{3}=0.128 \mathrm{eb}$; these values are kept constants for all isotopes. The IBM-2 CM values are tabulated in Table 3 . The $B(E 2)$ values of $\mathrm{Po}$ increase smoothly with the neutron number. The $B\left(E 2 ; 2_{1}^{+} \rightarrow 0_{1}^{+}\right)$and $B\left(E 2 ; 4_{1}^{+} \rightarrow 2_{1}^{+}\right)$values are of the same behavior which is typically increasing toward the end of the 
shell. The transition $B\left(E 2 ; 2_{2}^{+} \rightarrow 2_{1}^{+}\right)$is weak and has a small value because of the M1 mixing. The values of $B\left(E 2 ; 2_{3}^{+} \rightarrow 2_{1}^{+}\right)$are small, bacause it is the transition from the mixed symmetric state $2_{3}^{+}$to the symmetric state $2_{1}^{+}$, i.e., the transition from the gamma band to $g s$-band. The quadrupole moment for the excited first state $Q\left(2_{1}^{+}\right)$is given in Table 3 . It is seen that these values are positive. This indicates that the Po isotopes take an oblate shape in $2_{1}^{+}$states. The values of $Q\left(2_{1}^{+}\right)$decrease smoothly, as the neutron number increases, because the nucleus acquires the spherical shape, by approaching the closed shell $N=126$.

\section{Conclusions}

We have studied the nuclear structure and the reduced probability of the electrical transitions $B(E 2)$ and $Q\left(2_{1}^{+}\right)$in ${ }^{194-204} \mathrm{Po}$ isotopes within the Interacting Boson Model Configuration Mixing (IBM-2 $\mathrm{CM}$ ). The polonium isotopes in the normal configuration $N_{\pi}=1$ take a vibrational shape (near spherical shape), i.e., they are characterized by the U(5) symmetry. Based on the results concerning the energy states, we considered normal excitations of the configuration $N_{\pi}=1$, as well as the intruder excitations for $N_{\pi}=3$. The systematic is locally disturbed by the mixing of the regular and intruder configurations. This becomes even more clear, when looking at the $I=2$ multiple involving the ${ }^{184-192}$ Os isotopes $\left(N_{\pi}=3\right)$ in the regular or normal configuration and ${ }^{192-204}$ Po isotopes $\left(N_{\pi}=3\right)$ intruder excitations. In Table (1), Po isotopes in $N_{\pi}=1$ lie in the vibrational region near the spherical shape, and these isotopes tend to a deformed character in the intruder configuration $N_{\pi}=3$. The shape of Po isotopes is vibrational in the $N_{\pi}=1$ configuration. But, in the $N_{\pi}=3[(2 p-2 h)$ across the major shell] configuration, they take a rotational shape like Os nuclei. In the present research, we performed a detailed analysis of the ${ }^{194-204}$ Po series of isotopes using the IBM-2, involving double boson excitations through the protonclosed shell (called the intruder configuration zone) and their contact with the normal configuration space within the IBM-2 CM. We have identified the Hamiltonian and the $E 2$ operator describing this interacting boson system and have made a least-square fit to the known experimental data.

In addition, we have evaluated the effective charges for bosons which are normalized to the experimental value of $B\left(E 2 ; 2_{1}^{+} \rightarrow 0_{1}^{+}\right)$. For simplicity, we use $e_{\pi}=e_{v}=e_{1}$, where $e_{1}=0.29 e b$ and $e_{3}=0.128 e b$; these values are kept constants for all isotopes. The electric transition probabilities $B(E 2)$ are given in Table 3. The values of quadrupole moments for the first excited state decrease smoothly, as the neutron number increases. This is due to approaching the closed shell $N=126$; i.e., the shape of a Po nucleus become close to spherical.

1. J.L. Wood, K. Heyde, W. Nazarewic, M. Huyse, P. van Duppen. Coexistence in even-mass nuclei. Phys. Rep. 215, 101 (1992).

2. G.D. Dracoulis, B. Fabricius, A.E. Stuchbery, A.O. Macchiavelli, W. Korten, F. Azaiez, E. Rubel, M.A. Deleplanque, R.M. Diamond, F.S. Stephens. Shape coexistence from the structure of the yrast band in ${ }^{174} \mathrm{Pt}$. Phys. Rev. C 44 (4), R1246 (1991).

3. K. Elliott, S.F. King, P.L. White. Unification constraints in the next-to-minimal supersymmetric standard model. Phys. Lett. B 351, 213 (1995).

4. M.P. Carpenter, R.V.F. Janssens, H. Amro, D.J. Blumenthal, L.T. Brown, D. Seweryniak, P.J. Woods, D. Ackermann, I. Ahmad, C. Davids, S.M. Fischer, G. Hackman, et al. Excited states in ${ }^{176,178} \mathrm{Hg}$ and shape coexistence in very neutron-deficient $\mathrm{Hg}$ isotopes. Phys. Rev. Lett. 78, 19, 178 (1997).

5. M. Abed Al-Jubbori. Investigation of energy levels and electromagnetic transitions for Yb-Pt nuclei with $N=108$ using IBM, IVBM, and BMM. Ukr. J. Phys., 62 (11), 936 (2017).

6. A.M. Oros, K. Heyde, C. De Coster, B. Decroix, R. Wyss, B.R. Barrett, P. Navratil. Shape coexistence in the light Po isotopes. Nucl. Phys. A 645, (1) 107 (1999).

7. J.E. García-Ramos, K. Heyde. Nuclear shape coexistence in Po isotopes: An interacting boson model study. Phys. Rev. C 92, 3, 034309 (2015).

8. F. Iachello, A. Arima. The Interacting Boson Model (Cambridge University Press, 1987).

9. H. Tagziria, M. Elahrash, W.D. Hamilton, M. Finger, J. John, P. Malinsky, V.N. Pavlov. The role of the $C_{L}^{\rho}$ parameters in IBM-2 as exemplified by the nuclear structure of ${ }^{152}$ Gd. J. Phys. G. Nucl. Part. Phys. 16 (9), 1323 (1990).

10. P.D. Duval, B.R. Barrett. Configuration mixing in the interacting boson model. Phys. Lett. B 100 (3), 223 (1981).

11. P.D. Duval, B.R. Barrett. Quantitative description of configuration mixing in the interacting boson model. Nucl. Phys. A 376 (2), 213 (1982).

12. R. Fossion, K. Heyde, G. Thiamova, P. Van Isacker. Completely compensated Intruder bands and configuration mixing in lead isotopes. Phys. Rev. C 67 (2), 024306 , (2003).

13. M. Sambataro, G. Molnár. Configuration mixing in Mo isotopes. Nucl. Phys. A 376 (2), 201 (1982).

ISSN 2071-0194. Ukr. J. Phys. 2021. Vol. 66, No. 7 
14. A. Bohr, B.R. Mottelson. Nuclear Structure: Volume 1 (W.A. Benjamin, 1969).

15. A.F. Barfield, B.R. Barrett, K.A. Sage, P.D. Duval. The interacting Boson Model applied to the mercury isotopes. Z. Phys. A - Atoms Nucl. 311 (3), 205 (1983).

16. B. Decroix, J. De Beule, C. De Coster, K. Heyde, A.M. Oros, P. Van Isacker. Particle-hole excitations in the interacting boson models IBM-2 and IBM-3. Phys. Rev. C 57 (5), 2329 (1998).

17. R.B. Firestone. Nuclear data sheets for $A=184$. Nucl. Data Sheets 58 (1), 243 (1989).

18. B. Singh. Nuclear data sheets for $A=188$. Nucl. Data Sheets 59 (1), 133 (1990).

19. B. Singh. Nuclear data sheets for $A=190$. Nucl. Data Sheets 61 (2), 243 (1990).

20. V.S. Shirley. Nuclear data sheets for $A=192$. Nucl. Data Sheets 64, 205 (1991).

21. C.M. Baglin. Nuclear data sheets for $A=186$. Nucl. Data Sheets, 82 (1), 1 (1997).

22. W.D. Hamilton, A. Irbäck, J.P. Elliott. Mixed-symmetry interacting-boson-model states in the nuclei ${ }^{140} \mathrm{Ba},{ }^{142} \mathrm{Ce}$, and ${ }^{144} \mathrm{Nd}$ with $N=84$. Phys. Rev. Lett. 53 (26), 2469 (1984).

23. M.A.H. Muhi, S.N. Abood. Configuration mixing in Pt nuclei within interacting boson model-2. J. Appl. Math. Phys. 8 (3), $492(2020)$
24. W. Younes, J.A. Cizewski. Systematical behavior of even- $A$ polonium isotopes. Phys. Rev. C 55 (3), 1218 (1997).

25. C.M. Lederer, V.S. Shirley. Table of Isotopes (Wiley, 1978)

26. R.B. Firestone, V.S. Shirley. Table of Isotopes (Wiley, 1998).

Received 03.04.20

Л.А. Наджам, С.Н. Абуд, Ф.М.А. Ель-Джомейлі

ЗМІШУВАННЯ КОНФІГУРАЦІЙ ДЛЯ ІЗОТОПІВ Ро В МОДЕЛІ-2 ВЗАЄМОДІЮЧИХ БОЗОНІВ

Аналізується послідовність ізотопів ${ }^{194-204}$ Ро в рамках Моделі-2 Взаємодіючих Бозонів зі змішуванням конфігурацій. Параметри гамільтоніана знайдено шляхом підгонки за методом найменших квадратів по відомих рівнях енергії, константам швидкості електричних переходів $B(E 2)$ і квадрупольним моментам $Q\left(2_{1}^{+}\right)$перших збуджених станів. Отримано добре узгодження з експериментом для всіх розглянутих величин. Зроблено висновок, що властивість співіснування форм є прихованою для ізотопів Ро, як і для ізотопів Os i Pt.

Ключові слова: Модель-2 Взаємодіючих Бозонів, змішування конфігурацій, $B(E 2), Q\left(2_{1}^{+}\right)$. 